\title{
Associações entre autoavaliações negativas de saúde e vulnerabilidade macroestrutural, social e de saúde em idosos: Estudo Fibra, Campinas, Brasil
}

\author{
Associations between negative self-rated health and macro-structural, social and \\ health vulnerability in elderly people: Fibra Study, Campinas, Brazil
}

\section{Anita Liberalesso Neri ${ }^{a}$, Monica Sanches Yassuda ${ }^{b}$, André Fattoric, Maria Elena Guarientod, Flávia Silva Arbex Borime}

\footnotetext{
a Programa de Pós-Graduação em Gerontologia da Universidade Estadual de Campinas (Unicamp). Coord. do Estudo Fibra, polo Unicamp. <anitalbn@fcm.unicamp.br> b Programa de Pós-Graduação em Gerontologia da Universidade Estadual de Campinas (Unicamp). <monica@usp.br>

Programa de Pós-Graduação em Gerontologia da Universidade Estadual de Campinas (Unicamp). <afattori@unicamp.br>

d Programa de Pós-Graduação em Gerontologia da Universidade Estadual de Campinas (Unicamp). <meguar@fcm.unicamp.br>

e Programa de Pós-Graduação em Gerontologia da Universidade Estadual de Campinas (Unicamp). Revisão e redação final do manuscrito. <flarbex@hotmail.com>
}

\section{ARTICLE INFO}

\section{Article history}

Received: 10/06/2015

Accepted: $27 / 07 / 2015$

Correspondent Author

Anita Liberalesso Neri

PPG em Gerontologia - Faculdade de

Ciências Médicas - Unicamp

Av. Tessália Vieira de Camargo, 126

Cidade Universitária

13083-887 Campinas, SP, Brasil

<anitalbn@fcm.unicamp.br>

(C) 2014 All rights reserved

\section{Editors}

Alfredo Cataldo Neto

Irenio Gomes

\begin{abstract}
RESUMO
Objetivo: Investigar associações entre avaliações negativas de saúde e indicadores de vulnerabilidade estrutural (acesso e uso de serviços médicos, hospitalares e odontológicos e consumo de medicamentos), social (sexo, idade, escolaridade, renda pessoal, arranjos de domicílio, expectativa de cuidado e envolvimento social), e de saúde (doenças crônicas, sinais e sintomas, problemas funcionais para alimentação, risco cardiovascular, fragilidade, sintomas depressivos e status cognitivo), em idosos brasileiros recrutados na comunidade. Métodos: Participaram 2.597 idosos (65,7\% mulheres, com 65 anos e mais $(M=72,4 \pm 5,6)$, sem déficit cognitivo sugestivo de demência, integrantes de amostras probabilísticas de 7 cidades brasileiras selecionadas por conveniência. As variáveis de interesse foram avaliadas por meio de medidas de autorrelato, antropométricas e de desempenho. Resultados: Análise multivariada de regressão mostrou associações significantes $(p \leq 0,05)$ entre autoavaliação negativa de saúde e 3 ou mais sinais e sintomas $(O R=2,42), 3$ ou mais problemas funcionais para alimentação $(O R=2,30)$, renda $\leqq 3 \mathrm{SM}(\mathrm{OR}=2,17)$, não ter ido ao dentista no ano anterior $(\mathrm{OR}=2,09)$, consumo de 3 ou mais remédios $(O R=2,02)$, 3 ou mais doenças crônicas $(O R=2,00)$, analfabetismo $(O R=1,58), 4$ ou mais noites de hospitalização $(O R=1,17)$ e uso de serviços públicos de saúde $(\mathrm{OR}=1,06)$. Conclusões: Em interação múltipla, indicadores de vulnerabilidade estrutural, social e em saúde criam um círculo vicioso de influências deletérias à qualidade de vida dos idosos, que tendem a se refletir em autoavaliações negativas de saúde.

DESCRITORES: Saúde do idoso; Autopercepção; Idoso, fatores socioeconômicos; Vulnerabilidade em saúde; Condições de vida.
\end{abstract}

\begin{abstract}
Purpose: This study was aimed at investigating associations between negative self-rated health and macro-structural (access and use of medical, hospitalar and odontological services, as well as consumption of medicines), social (sex, age, schooling, individual income, living arrangements, expectancy of care, and social participation), and health indicators (chronic diseases, signals and symptoms, functional problems to feeding, cardiovascular risk, frailty, depressive symptoms, and cognitive status) in Brazilian elderly recruited in the community. Methods: Participants were 2.597 seniors (65,7\% women), aged 65 and above $(M=72,4 \pm 5,6)$, without cognitive deficit suggestive of dementia. They integrated the probabilistic samples from 7 Brazilian cities selected by convenience. The variables were assessed by self-report, anthropometric and performance instruments and measures. Results: Multivariate analyses of regression showed significant associations $(p \leq 0,05)$ between self-rated health and having 3 or more signals and symptoms $(O R=2,42), 3$ or more functional problems to feeding $(O R=2,30)$, individual income $\leq 3 \mathrm{MW}(\mathrm{OR}=2,17)$, no visits to the dentist in the last year $(O R=2,09)$, consumption of 3 or more medicines $(O R=2,02), 3$ or more chronic illnesses $(O R=2,00)$, illiteracy $(O R=1,58)$, more than 4 nights of hospitalization $(O R=1,17)$, and use of public health services $(O R=1,06)$. Conclusions: In multiple interaction, macro-structural, social and health indicators of vulnerability can generate a vicious cycle of deleterious influences over old people's quality of life, which tend to reflect in negative self-rated health

KEYWORDS: Aging health; Self-rated health; Aged, socioeconomic factors; Health vulnerability; Living conditions.
\end{abstract}




\section{INTRODUÇÃO}

A autoavaliação de saúde é um construto valorizado por investigações e por sistemas de vigilância epidemiológica ${ }^{1,2}$, porque prediz desfechos como mortalidade, morbidade e incapacidade ${ }^{3,4,5}$ e participa da explicação de outros, tais como autocuidado, ${ }^{6}$ estilo de vida ${ }^{7}$ e bem estar subjetivo., ${ }^{7,8}$ A resposta é baseada num processo cognitivo de comparação entre as experiências fisiológicas, emocionais e sociais do avaliador, que leva em conta critérios e expectativas individuais e sociais a respeito do que é uma boa saúde numa dada idade. ${ }^{9}$

Dependendo de seus objetivos, inquéritos de saúde e pesquisas epidemiológicas ora atribuem função preditora, ${ }^{1,3,4}$ ora lidam com a autoavaliação de saúde como variável dependente $\mathrm{e}^{5,6,10-12}$. Existe farta literatura sobre a relação entre autoavaliação negativa de saúde na velhice e risco para mortalidade, em associação com sexo, renda, escolaridade, comportamentos de saúde e doenças crônicas ${ }^{1,3,4}$. De modo geral, os mais pobres e menos educados têm pior percepção da própria saúde e mais doenças crônicas e incapacidades, principalmente se forem mulheres. Outras pesquisas sugerem que a relação entre autoavaliação negativa da saúde e mortalidade, na velhice, é mediada por altos níveis de incapacidade e por multimorbidades 5 . Por outro lado, são bem disseminados dados de pesquisas sobre determinantes socioeconômicos da autoavaliação negativa de saúde, entre eles a desigualdade social e a pobreza em interação com idade ${ }^{13}$ e gênero, ${ }^{14} \mathrm{com}$ a escassez de serviços bem qualificados de atenção à saúde ${ }^{15-19,}$, com apoios familiares e sociais deficitários, ${ }^{20,21}$ fragilidade ${ }^{22}$ e da depressão. ${ }^{23}$

A complexidade do fenômeno é um estímulo à adoção de modelos complexos para análise desse construto latente. Essa é a posição adotada pela presente pesquisa, que considera associações entre autoavaliações negativas de saúde e indicadores de vulnerabilidade macroestrutural, social e de saúde em idosos. Vulnerabilidade é um processo dinâmico de adaptação negativa às adversidades e aos riscos pontuais ou cumulativos da velhice e do curso de vida $^{24}$. Fatores de risco são características mensuráveis de indivíduos ou de seu ambiente que predizem desfechos negativos num critério específico. ${ }^{24}$ Refletem-se no fracasso da resiliência ou no aumento da vulnerabilidade ou da susceptibilidade a estressores de origem macroestrutural, lastreados nas políticas sociais e no nível de desenvolvimento socioeconômico das nações (ex.: serviços de saúde insuficientes ou de baixa qualidade), aos estressores associados a desvantagens sociais que afetam a adaptação e o desenvolvimento individual (exs: baixa escolaridade, pobreza e exposição a desastres ambientais) e aos relacionados à fragilização da saúde física e mental (exs: comorbidades, incapacidade física e depressão). ${ }^{24,25}$

No âmbito internacional, existem numerosos estudos que contemplam a relação entre autoavaliação de saúde e um ou mais tipos de vulnerabilidade em idosos, como por exemplo o de Andrew e Keefe. ${ }^{25}$ No Brasil discute-se o conceito, ${ }^{26,27}$ publicam-se pesquisas metodológicas ${ }^{28}$ e observacionais de corte transversal e de base populacional ${ }^{26}$ envolvendo a noção de tipos e níveis de vulnerabilidade em relação a diversos desfechos objetivos e subjetivos de saúde.

\section{OBJETIVO}

Investigar relações entre avaliações negativas de saúde e indicadores de vulnerabilidade de natureza macroestrutural, social e de saúde, em idosos recrutados na comunidade.

\section{MÉTODOS}

\section{Participantes}

Foram 2.597 idosos sem déficit cognitivo sugestivo de demência, integrantes de amostras representativas da população de 65 anos e mais, de sete cidades brasileiras escolhidas por conveniência (Campinas, SP; Belém, PA; Campina Grande, PB; Parnaíba, PI; Poços de Caldas, MG; Distrito de Ermelino Matarazzo em São Paulo, SP e Ivoti, RS), no contexto de um estudo multicêntrico de corte transversal sobre fragilidade. ${ }^{29} \mathrm{O}$ Estudo da Fragilidade em Idosos Brasileiros, acrônimo Fibra, envolveu uma rede composta por pesquisadores de quatro universidades (USP Ribeirão Preto, UFMG, UERJ e Unicamp), referidas como polos.

O recrutamento foi realizado em domicílios familiares. Idosos com 65 anos e mais que residiam permanentemente nos setores censitários e respectivas residências foram considerados elegíveis. Os critérios de exclusão foram: presença de déficits sensoriais, psicomotores, cognitivos e de comunicação prejudiciais à participação; sequelas graves de acidente vascular encefálico (AVE); doença de Parkinson em estágio avançado; restrição permanente ou temporária ao leito; estar em estágio terminal ou ter câncer (exceto de pele). ${ }^{30}$

Os idosos selecionados eram convidados para uma sessão de coleta de dados, realizada em locais públicos de fácil acesso, durante um período de 40 a 120 minutos. Os participantes foram esclarecidos sobre os objetivos, o caráter voluntário da participação, o direito a sigilo e a ausência de riscos físicos e psicológicos e assinaram um 
termo de consentimento livre e esclarecido aprovado pelo Comitê de Ética da Faculdade de Ciências Médicas da Unicamp, sob o parecer no 207/2008 (CAAE 3954. 7104.0.1001.5454). Todos foram submetidos a um bloco de medidas demográficas e socioeconômicas, clínicas, antropométricas, de fragilidade ${ }^{30}$ e de rastreio cognitivo (Mini-Exame do Estado Mental - MEEM). Pontuação inferior à nota de corte ajustada pelos anos de escolaridade, menos um desvio-padrão foi utilizada como critério de exclusão de 881 idosos. Estes não participaram do segundo bloco de medidas de saúde, funcionalidade e psicossociais, entre elas as de interesse para este projeto ${ }^{31}$.

\section{Variáveis e medidas}

1. Autoavaliação de saúde. Pediu-se aos idosos que classificassem a qualidade da própria saúde, com base numa escala de 5 pontos em que $1=$ muito ruim, $2=$ ruim, $3=$ regular, $4=$ boa e $5=$ muito boa ${ }^{9}$

2. Indicadores de vulnerabilidade estrutural. Serviços médicos e odontológicos mais utilizados pelos idosos (públicos $\times$ privados); número de consultas médicas e odontológicas nos 12 meses anteriores; consumo de medicamentos nos últimos 3 meses e maior número de pernoites em hospital nos últimos 12 meses.

3. Indicadores de vulnerabilidade social. Sexo, idade, alfabetização, número de anos de escolaridade, renda pessoal, arranjo domiciliar, expectativa de cuidado e participação em atividades avançadas de vida diária (AAVD). Foram colhidos valores brutos de renda, depois transformados em unidades e em faixas de salários mínimos. $\mathrm{O}$ arranjo domiciliar foi indicado pelas respostas sozinho, com cônjuge, com descendentes ou com o cônjuge e descendentes à pergunta "com quem mora?". O envolvimento em AAVD correspondeu a um inventário com 13 itens relativos à sociabilidade (ex.: fazer visitas), à produtividade (ex.: trabalho) e à participação social (ex.: fazer parte de conselhos). ${ }^{32}$ Foi calculado o percentual de AAVD abandonadas por cada idoso e estabelecidas faixas considerando-se os tercis da distribuição desses valores.

4. Indicadores de vulnerabilidade em saúde. Incluíram medidas de fragilidade, doenças crônicas, risco cardiovascular, sinais e sintomas, problemas funcionais para alimentação, sintomas depressivos e nível cognitivo. A fragilidade foi operacionalizada pelos critérios inatividade física, perda de peso não intencional, fadiga, baixa força de preensão e lentidão da marcha. Foram considerados como frágeis os idosos que pontuaram em 3 ou mais critérios, como pré-frágeis os que pontuaram em 1 ou 2 e como não frágeis os que não pontuaram em nenhum. ${ }^{29,30}$ Foi computado o número de diagnósticos médicos, concernentes a doenças crônicas (cardiopatias, hipertensão arterial, diabetes, doença cerebrovascular, tumor/câncer, artrite, pneumopatias, depressão e osteoporose), assim como foram contados os sinais e sintomas (entre incontinência urinária, incontinência fecal, perda de apetite, perda de peso, ganho de peso, problemas de memória, quedas, tristeza e problemas de sono) e os problemas funcionais relativos à mastigação e à deglutição, todos tomando-se como base os últimos 12 meses e o relato dos idosos ${ }^{29}$. Risco cardiovascular foi referenciado à medida da relação cintura-quadril ${ }^{33}$. A Escala de Depressão Geriátrica (EDG) ${ }^{34}$ foi usada para rastreio de sintomas, com nota de corte $\geq 6$. A pontuação no MEEM foi dividida nas faixas $<23$ (baixa); 24 a 26 (intermediária) e > 26 (alta) pontos.

\section{Análise de dados}

Foram comparadas as características dos idosos com autoavaliação positiva (muito bom, bom e regular) e as dos idosos com autoavaliação negativa (ruim e muito ruim) de saúde. A significância estatística das diferenças observadas foi avaliada por meio do teste qui-quadrado de Pearson. Nos mesmos grupos, as variáveis ordinais tiveram suas distribuições comparadas pelo teste de Mann-Whitney. Análise de regressão logística univariada avaliou a magnitude das associações entre cada um dos indicadores de vulnerabilidade e autoavaliação negativa de saúde, por meio de odds ratio $(O R)$ e respectivos intervalos de confiança de $95 \%$. Em seguida foi realizada análise de regressão logística multivariada, modelo hierárquico com critério stepwise envolvendo três modelos. No modelo 1 foi calculada a associação múltipla de todos os indicadores de vulnerabilidade macroestrutural. No modelo 2 foram incluídos os indicadores de vulnerabilidade macroestrutural cujas associações com a variável dependente alcançaram nível de significância $\geq 0,05$ e todos os indicadores de vulnerabilidade social. No modelo 3 entraram as variáveis dos domínios macroestrutural e social com associação estatisticamente significativa com autoavaliação de saúde e todos os indicadores de vulnerabilidade em saúde. No resultado final foram retidos os indicadores ajustados que mantiveram associação estatisticamente significante $(\mathrm{p} \leq 0,05)$ com autoavaliação de saúde.

\section{RESULTADOS}

Entre os 2.597 idosos, 65,7\% eram mulheres. A idade média foi 72,5 55,6 anos; a média de anos de escolaridade, $4,7 \pm 4,1$, e a renda média, 2,5 salários mínimos $(\mathrm{dp}=3,4) ; 22,5 \%$ eram analfabetos. Da amostra total, 1.893 idosos avaliaram positivamente 
a própria saúde (muito boa, boa e regular) e 704 (27,1\%) avaliaram-na negativamente (ruim e muito ruim). Em comparação com os que avaliaram positivamente a própria saúde, idosos com autoavaliação negativa foram significantemente mais vezes ao médico, menos vezes ao dentista e consumiam maior número de medicamentos; tinham menos anos de escolaridade, menor renda pessoal e maior percentual de AAVD abandonadas.

Tabela 1. Comparações entre os participantes com avaliação positiva e com avaliação negativa de saúde, conforme os indicadores de vulnerabilidade. Estudo Fibra, polo Unicamp, Brasil. Idosos, 2008-2009.

\begin{tabular}{|c|c|c|c|c|c|c|c|c|}
\hline \multirow{2}{*}{ Vulnerabilidade } & \multirow{2}{*}{ Indicadores } & \multicolumn{3}{|c|}{ Com autoavaliação positiva de saúde } & \multicolumn{3}{|c|}{ Com autoavaliação negativa de saúde } & \multirow{2}{*}{$\mathbf{p}$} \\
\hline & & $\mathbf{n}$ & Média & dp & $\mathbf{n}$ & Média & dp & \\
\hline \multirow[t]{4}{*}{ Macroestrutural } & Consultas médicas/12 meses & 1791 & 4,30 & 4,46 & 677 & 4,91 & 5,59 & 0,016 \\
\hline & Consultas odontológicas/12 meses & 1863 & 1,01 & 2,26 & 695 & 0,59 & 1,63 & $<0,001$ \\
\hline & Internações hospitalares/12 meses & 234 & 10,94 & 52,92 & 147 & 10,84 & 19,79 & 0,190 \\
\hline & Medicamentos/ 3 meses & 1868 & 2,36 & 2,19 & 697 & 2,96 & 2,39 & $<0,001$ \\
\hline \multirow[t]{4}{*}{ Social } & Idade & 1893 & 72,50 & 5,63 & 704 & 72,07 & 5,40 & 0,127 \\
\hline & Anos de escolaridade & 1891 & 4,73 & 4,12 & 702 & 3,47 & 3,49 & $<0,001$ \\
\hline & Renda pessoal em SM & 1833 & 2,56 & 3,43 & 692 & 1,75 & 1,94 & $<0,001$ \\
\hline & AAVD abandonadas (\%) & 1877 & 36,13 & 23,06 & 702 & 39,53 & 25,13 & 0,002 \\
\hline \multirow[t]{6}{*}{ Em saúde } & Doenças crônicas/12 meses & 1891 & 2,03 & 1,39 & 703 & 2,75 & 1,57 & $<0,001$ \\
\hline & $\mathrm{RCQ}$ & 1872 & 0,91 & 0,08 & 698 & 0,91 & 0,08 & 0,919 \\
\hline & Sinais e sintomas $/ 12$ meses & 1892 & 2,44 & 1,69 & 703 & 3,56 & 1,74 & $<0,001$ \\
\hline & Probl. func. Alimentação/12 meses & 1781 & 1,42 & 1,65 & 549 & 2,39 & 2,03 & $<0,001$ \\
\hline & Escore na EDG & 1874 & 3,16 & 2,50 & 701 & 4,55 & 2,89 & $<0,001$ \\
\hline & Escore no MEEM & 1893 & 25,22 & 3,03 & 704 & 24,09 & 3,15 & $<0,001$ \\
\hline
\end{tabular}

Tabela 2. Comparação entre as frequências de avaliação positiva e negativa de saúde e resultados da análise de regressão logística univariada da associação entre autoavaliação negativa de saúde e indicadores de vulnerabilidade macroestrutural. Estudo Fibra, polo Unicamp, Brasil. Idosos, 2008-2009.

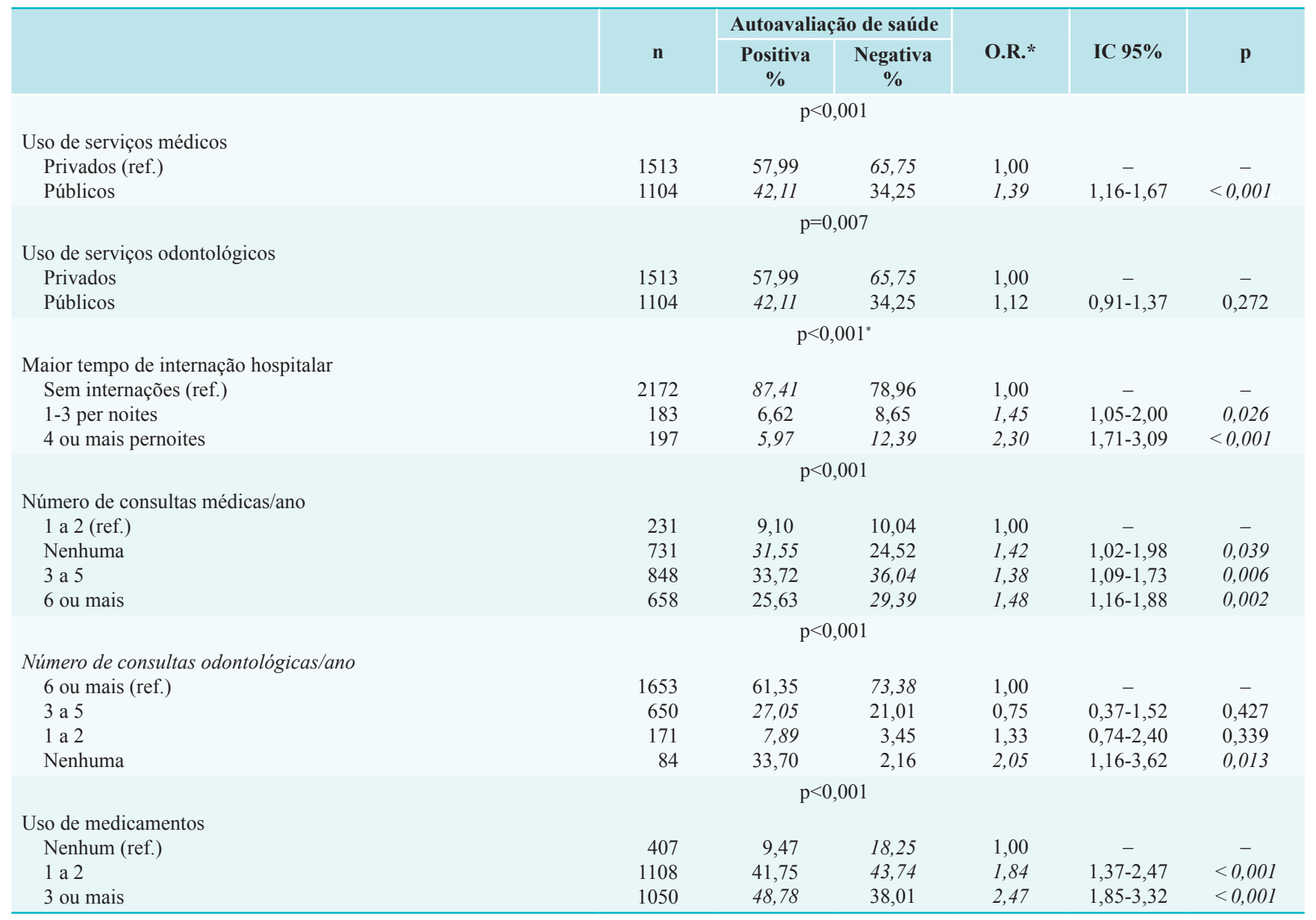


Apresentaram maior número de doenças, mais sinais e sintomas, mais problemas funcionais para alimentação, mais sintomas depressivos e menor a pontuação no MEEM (Tabela 1).

Houve um percentual significantemente mais alto de idosos com autoavaliações negativas de saúde entre os que se valiam de serviços públicos de saúde, os que foram hospitalizados por mais tempo, os que não haviam ido ao dentista no ano anterior e os que consumiam 3 ou mais medicamentos. Ausência de consultas médicas e 3 ou mais consultas apresentaram associação estatisticamente significante com autoavaliação negativa de saúde (Tabela 2).

Os idosos com renda até 5 salários mínimos, 8 anos ou menos de escolaridade formal e os analfabetos foram os que apresentaram chances significantemente maiores de avaliar negativamente a própria saúde. Sexo feminino, maior taxa de abandono de AAVD e residência com descendentes também se associaram com a variável de interesse (Tabela 3 ).

Tabela 3. Comparação entre as frequências de avaliação positiva e negativa de saúde e resultados da análise de regressão logística univariada da associação entre autoavaliação negativa de saúde e indicadores de vulnerabilidade social. Estudo Fibra, Brasil. Idosos, 2008-2009.

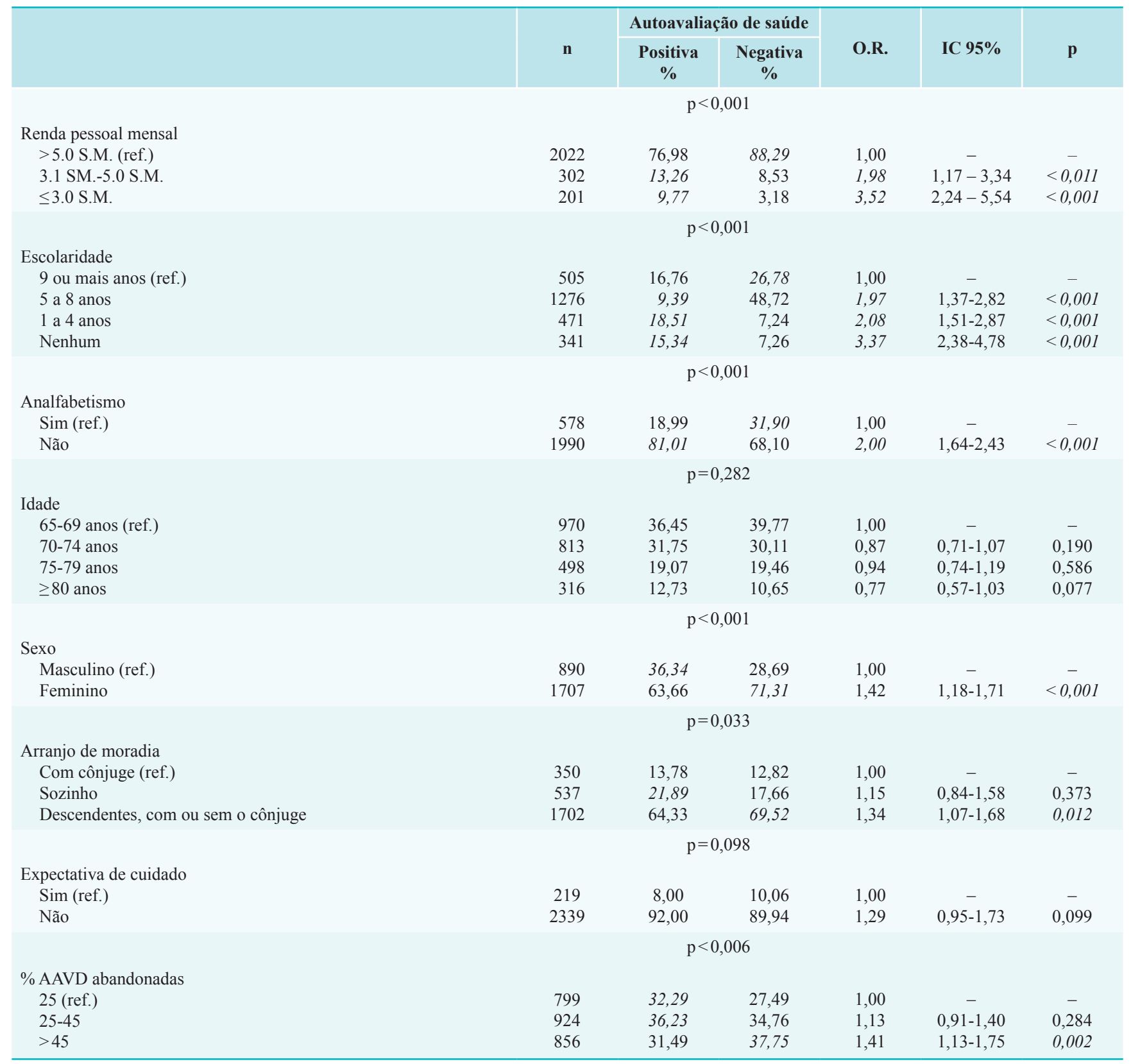


Entre os indicadores vulnerabilidade em saúde, as associações foram com maior número de sinais e sintomas, de problemas funcionais para a alimentação e de doenças crônicas; pontuação $\geq 6$ na EDG, pontuar para pré-fragilidade/fragilidade e baixa pontuação no MEEM (Tabela 4).

A análise de regressão hierárquica multivariada testou três modelos. No modelo 1, que incluiu todos os indicadores de vulnerabilidade estrutural, associaram-se com autoavaliação negativa de saúde: consumo de medicamentos, não ter ido a nenhuma consulta odontológica no ano anterior, maior número de internações hospitalares nos últimos 12 meses e uso de serviços públicos de saúde. No modelo 2, que incluiu os indicadores de vulnerabilidade social, foram observadas associações com renda $\leq 3 \mathrm{SM}$ e analfabetismo. No modelo 3, a estimativa das associações com os indicadores de vulnerabilidade em saúde ajustados pelos indicadores dos dois outros domínios resultou em associações estatisticamente significantes com 3 ou mais doenças crônicas, 1 ou mais sinais e sintomas, pontuação $\geq 6$ na EDG e 1 ou mais problemas funcionais para alimentação (Tabela 5).

Tabela 4. Comparação entre as frequências de avaliação positiva e negativa de saúde e resultados da análise de regressão logística univariada da associação entre autoavaliação negativa de saúde e indicadores de vulnerabilidade em saúde. Estudo Fibra, Brasil. Idosos, 2008-2009.

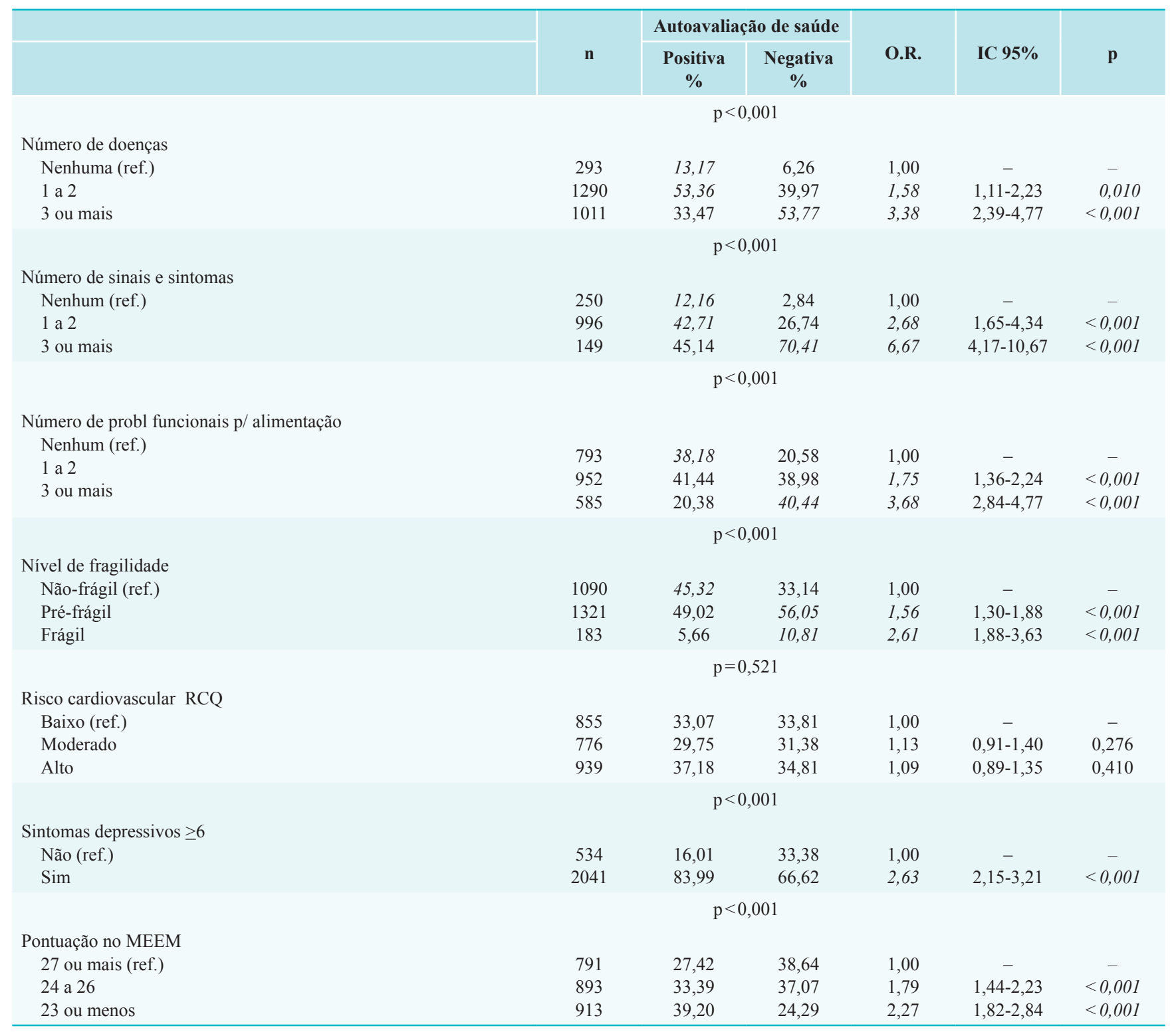


Tabela 5. Resultados da análise de regressão hierárquica das associações múltiplas entre autoavaliação negativa de saúde e indicadores de vulnerabilidade macroestrutural, social e em saúde. Estudo Fibra, Brasil. Idosos, 2008-2009.

\begin{tabular}{|c|c|c|c|c|c|c|c|c|c|}
\hline & \multicolumn{3}{|c|}{ Modelo 13} & \multicolumn{3}{|c|}{ Modelo 2} & \multicolumn{3}{|c|}{ Modelo 3} \\
\hline & OR & $\begin{array}{c}\text { IC } 95 \% \\
\text { OR }\end{array}$ & p & OR & $\begin{array}{c}\text { IC } 95 \% \\
\text { OR }\end{array}$ & $\mathbf{p}$ & OR & $\begin{array}{c}\text { IC } 95 \% \\
\text { OR }\end{array}$ & $\mathbf{p}$ \\
\hline \multicolumn{10}{|l|}{ Uso medicamentos } \\
\hline Nenhum (ref.) & 1,00 & - & - & 1,00 & - & - & 1,00 & - & - \\
\hline 1 a 2 & 1,96 & $1,32-2,92$ & $<0,001$ & 1,96 & $1,31-2,93$ & 0,001 & 1,61 & $1,05-2,45$ & 0,028 \\
\hline 3 ou mais & 2,91 & $1,96-4,33$ & $<0,001$ & 3,02 & $2,02-4,51$ & $<0,001$ & 2,02 & $1,31-3,12$ & 0,002 \\
\hline \multicolumn{10}{|l|}{ Cons. odontológicas } \\
\hline 6 ou mais (ref.) & 1,00 & - & - & 1,00 & - & - & 1,00 & - & - \\
\hline 3 a 5 & 0,83 & $0,32-2,12$ & 0,689 & 0,77 & $0,30-1,99$ & 0,587 & 0,69 & $0,26-1,80$ & 0,444 \\
\hline 1 a 2 & 1,95 & $0,90-4,25$ & 0,092 & 1,73 & $0,79-3,79$ & 0,169 & 1,68 & $0,76-3,73$ & 0,202 \\
\hline Nenhuma & 2,63 & $1,23-5,62$ & 0,013 & 2,15 & $1,01-4,64$ & 0,049 & 2,09 & $0,96-4,57$ & 0,064 \\
\hline \multicolumn{10}{|l|}{ Uso serviços médicos } \\
\hline Privados (ref.) & 1,00 & - & - & 1,00 & - & - & 1,00 & - & - \\
\hline Públicos & 1,38 & $1,09-1,74$ & 0,007 & 1,08 & $0,84-1,38$ & 0,559 & 1,06 & $0,82-1,37$ & 0,678 \\
\hline \multicolumn{10}{|l|}{ Pernoites em hospital } \\
\hline Sem internação (ref.) & 1,00 & - & - & 1,00 & - & - & 1,00 & - & - \\
\hline 1 a 3 & 1,36 & $0,91-2,02$ & 0,134 & 1,26 & $0,84-1,90$ & 0,258 & 1,08 & $0,71-1,64$ & 0,735 \\
\hline 4 ou mais & 1,63 & $1,11-2,41$ & 0,014 & 1,53 & $1,03-2,27$ & 0,036 & 1,17 & $0,77-1,77$ & 0,457 \\
\hline \multicolumn{10}{|l|}{ Analfabetismo } \\
\hline Não (ref.) & & & & 1,00 & - & - & 1,00 & - & - \\
\hline Sim & & & & 1,86 & $1,44-2,41$ & $<0,001$ & 1,58 & $1,20-2,06$ & $<0,001$ \\
\hline \multicolumn{10}{|l|}{ Renda pessoal mensal } \\
\hline$>5.0 \mathrm{SM}$ (ref.) & & & & 1,00 & - & - & 1,00 & - & - \\
\hline $3.1-5.0 \mathrm{SM}$ & & & & 1,89 & $0,98-3,64$ & 0,057 & 1,65 & $0,85-3,22$ & 0,142 \\
\hline$\leq 3.0 \mathrm{SM}$ & & & & 2,61 & $1,45-4,70$ & 0,001 & 2,17 & $1,19-3,96$ & 0,011 \\
\hline \multicolumn{10}{|l|}{ Prob func alimentação } \\
\hline Nenhum (ref.) & & & & & & & 1,00 & $\stackrel{-}{103-186}$ & ${ }^{-}$ \\
\hline 1 a 2 & & & & & & & 1,39 & $1,03-1,86$ & $\begin{array}{l}0,029 \\
<0001\end{array}$ \\
\hline 3 ou mais & & & & & & & 2,30 & $1,67-3,16$ & $<0,001$ \\
\hline \multicolumn{10}{|l|}{ Sinais e sintomas } \\
\hline Nenhum (ref.) & & & & & & & 1,00 & - & - \\
\hline 1 a 2 & & & & & & & 1,85 & $1,04-3,28$ & 0,036 \\
\hline 3 ou mais & & & & & & & 2,42 & $1,36-4,30$ & 0,003 \\
\hline \multicolumn{10}{|l|}{ Doenças crônicas } \\
\hline Nenhuma (ref.) & & & & & & & 1,00 & - & - \\
\hline 1 a 2 & & & & & & & 1,40 & $0,85-2,31$ & 0,193 \\
\hline 3 ou mais & & & & & & & 2,00 & $1,19-3,36$ & 0,009 \\
\hline \multicolumn{10}{|l|}{ Sint depressivos $\geq 6$} \\
\hline Não (ref.) & & & & & & & 1,00 & - & - \\
\hline Sim & & & & & & & 1,41 & $1,07-1,86$ & 0,014 \\
\hline
\end{tabular}

\section{DISCUSSÃO}

Os indicadores de vulnerabilidade macroestrutural refletem variáveis de contexto sobre as quais os idosos não têm, ou têm limitado controle, visto que elas dependem de fatores macroeconômicos que interagem com as políticas e práticas sociais relativas à equidade em face dos grupos etários e do seu potencial produtivo. ${ }^{15-19}$ No modelo inicial da análise multivariada, eles foram representados por consumo de medicamentos, baixa frequência ou ausência de consultas odontológicas, maior duração de períodos de hospitalização e uso de serviços públicos de saúde. A ausência ou a baixa frequência de consultas odontológicas reflete a escassez e a inadequação de serviços públicos de atenção à saúde bucal, para atender adultos e idosos e, paralelamente, o alto custo dos serviços privados, que não podem ser pagos pela maioria. Espelha a dicotomia existente principalmente em países com forte desigualdade social, entre serviços públicos gratuitos para os muito pobres, e serviços privados pagos para os que têm recursos. ${ }^{15-19}$ Embora o público e o privado não sejam necessariamente definidores da qualidade dos serviços, na prática estes últimos resultam em maior eficácia, até 
mesmo porque as pessoas que deles se valem geralmente têm condições socioeconômica e de saúde melhores do que os primeiros. ${ }^{15-19}$ Resultam graves distorções que, no campo da saúde bucal concretizam-se em altos índices de edentulismo, de necessidade de próteses dentárias e de doenças bucais, ${ }^{35}$ condições que expõem os idosos ao risco de desenvolver doenças crônicas. Tal situação é menos grave no domínio da assistência médica. Mesmo assim, há precariedades quantitativas e qualitativas distribuídas de forma desigual pelo território e pelos vários segmentos da população. ${ }^{15} \mathrm{~A}$ escassez de oportunidades para diagnóstico e tratamento de médio e longo prazos tende a refletir-se na progressão de doenças para condições graves e críticas, que exigem internações custosas com grande chance de evoluir para dependência e morte.

O nível de renda e o de educação são características dos indivíduos, mas agregam outras que são relevantes no âmbito do grupo, tais como status conjugal, arranjo domiciliar e cuidado familiar, ${ }^{12,13,14}$ que se associam ao gênero ${ }^{14} \mathrm{e}$ à idade,,$^{13}$ ao nível de renda pessoal e da família' ${ }^{16,17}$ e ao nível de renda e de educação da vizinhança que, por sua vez, é preditor de bem estar subjetivo e objetivo dos idosos. ${ }^{7,8}$ Indicadores de renda e educação fornecem a estrutura mais próxima e necessária ao exercício de formas de sociabilidade espelhadas em arranjos de moradia, suporte material e instrumental em caso de doença, e envolvimento com atividades sociais complexas, que valorizam os idosos e seu capital social, facilitando o seu acesso a bens e a oportunidades sociais..$^{22,25,36}$ Em conjunto, esses fatores dependem de variáveis contextuais mais amplas, presentes na vida de mulheres e homens idosos. Entre elas estão as políticas previdenciárias e habitacionais e as de transporte, segurança e saúde, que criam condições diferenciais para resiliência ou para vulnerabilidade social dos diversos estratos da população e dos indivíduos.

Quatro mecanismos interrelacionados explicam a influência das variáveis macroestruturais e sociais sobre a saúde. Um é o estresse crônico associado à falta de prestígio social, cuja atuação sobre a saúde se dá por meio dos mecanismos de regulação hormonal e de seus efeitos sobre a função imune. ${ }^{38}$ Outro diz respeito a comportamentos de saúde, ${ }^{5,6}$ que incluem dieta, tabagismo, alcoolismo, exercícios físicos e consumo de medicamentos. Em terceiro lugar, podem ser citadas as influências materiais propriamente ditas, sob as formas de recursos financeiros, status social e contatos sociais. ${ }^{5,12,13,16,20,21}$ Em quarto lugar, atuam fatores psicológicos tais como senso de autoeficácia e estratégias de enfrentamento adaptativas. ${ }^{25}$
Os indicadores de vulnerabilidade em saúde traduzem a exposição dos idosos a doenças crônicas, sinais e sintomas, risco cardiovascular, fragilidade, depressão e baixo status cognitivo, resultantes das perdas normativas e não normativas associadas ao envelhecimento fisiológico. Estas não têm existência isolada, mas dependem da interação entre o organismo biológico e fatores contextuais atuais e do curso de vida. Nesta pesquisa, a frequência de queixas (sinais e sintomas, problemas de mastigação e deglutição e sintomas depressivos) explicou as autoavaliações negativas de saúde, sobrepondo-se a medidas de fragilidade e de obesidade abdominal traduzida em risco cardiovascular.

De forma sintética, é possível resumir os resultados obtidos da seguinte forma: idosos biológica e socialmente vulneráveis por efeito de fatores atuais e cumulativos provenientes dos contextos sociais distal e proximal são os que contaram e contam com menos apoio das instituições sociais encarregadas de proverlhes ajuda especializada para explicar e cuidar de suas queixas em saúde. ${ }^{15-18} \mathrm{Na}$ ausência de tratamentos preventivos e de acompanhamento na atenção básica e em casa, para muito idosos as situações de crise em saúde refletem-se em internação hospitalar. Na ausência de recursos materiais, parte dos idosos não tem acesso aos medicamentos de que necessitam, ${ }^{36}$ parte consome mais do que o necessário, ${ }^{38}$ outros seguem prescrições inadequadas ${ }^{39}$ e outros ainda, valem-se de automedicação. ${ }^{40}$ Não há contradição entre a associação entre o consumo de 3 ou mais medicamentos e autoavaliação negativa de saúde, encontrada na presente pesquisa e em outras que avaliaram o construto, ${ }^{36-40}$ pois o número de remédios que os idosos dizem que consomem reflete uma constelação de condições. Muitas vezes elas são conflitantes. Números, sejam eles grandes ou pequenos, não necessariamente significam atendimento suficiente e adequado às necessidades de saúde. Nesta pesquisa, o número de medicamentos explicou a variabilidade conjunta dos quatro indicadores de vulnerabilidade macroestrutural que permaneceram no modelo 1 , assim como covariou com os dois indicadores que permaneceram no modelo 2, no qual sexo, idade, renda, escolaridade, arranjo de moradia, expectativa de cuidado e envolvimento em AAVD tiveram sua variabilidade explicada por renda e escolaridade. A taxa observada de autoavaliações negativas de saúde foi comparável à obtida por estudos realizados fora do Brasil ${ }^{11,12,13,14}$ mas é pouco relevante notar similaridades, quando o mais importante é conhecer as variáveis que as prevalências.

Os dados sugerem novos encaminhamentos para a investigação da autoavaliação em saúde. Um deles é 
a realização de pesquisas longitudinais e prospectivas envolvendo amostras representativas dos vários segmentos econômicos e educacionais da população idosa, cujos dados devem ser submetidos a análises estatísticas avançadas. Outro é tornar mais sensível o instrumento de autoavaliação de saúde, tendo em mente a necessidade de saber o que os idosos levam em conta ao responder uma questão simples que pede que atribuam uma nota à sua saúde. Um terceiro é explorar o potencial de atuação da variável como mediadora de autocuidado e como moderadora dos sensos de controle e de autoeficácia em saúde. O quarto é a realização de estudos controlados de intervenção, envolvendo o uso da autoavaliação de saúde como variável dependente e como variável mediadora em vários contextos de saúde na velhice. Finalmente, é importante realizar pesquisas comparativas sobre a autoavaliação em saúde às idades, procurando separar efeitos das mudanças fisiológicas do envelhecimento e efeitos coorte, que repercutem na saúde e nos comportamentos dos idosos.

\section{AGRADECIMENTOS}

Agradecimentos a José Guilherme de Arruda Moura, Ludgleydson Fernandes de Araújo, Maria do Carmo Eulálio, Maria Eliane Catunda de Siqueira, Monica Sanches Yassuda e Geraldine Alves dos Santos, coordenadores do Estudo Fibra em Belém, PA; Parnaíba, PI; Campina Grande, PB; Poços de Caldas, MG; Distrito de Ermelino Matarazzo em São Paulo, SP, e Ivoti, RS. Também a Flávia Silva Arbex Borim, Stella Vital de Souza Torres e Deborah Dias da Silva, pela colaboração na coordenação da coleta de dados em Campinas, SP. Gratidão é devida a Juliana Martins Pinto e Natalia Oliveira Rodrigues pela coordenação da digitação dos dados, e a Efigênia Passareli Mantovani e Andrea Cristina Garofe Fortes Burgos pela ajuda no treinamento das equipes de cada cidade do polo Unicamp e no preparo do material da pesquisa.

Ao CNPq (processo no 555082/2006-7) e à FAEPEx Unicamp, pelo apoio financeiro.

\section{REFERÊNCIAS}

1. Lima-Costa MF, Peixoto SV, Matos DL et al. Predictors of 10-year mortality in a population of community-dwelling Brazilian elderly: the Bambuí Cohort Study of Aging. Cad. Saúde Pública. 2011;27 Sup 3:S360-S369.

2. Ministério da Saúde. [Internet]. Secretaria de Vigilância em Saúde. Secretaria de Gestão Estratégica e Participativa. Vigitel Brasil 2009: vigilância de fatores de risco e proteção para doenças crônicas por inquérito telefônico. Brasília: 2010 [acesso 2012 jun 23]. Disponível em: http://portal.saude.gov. br/portal/arquivos/pdf/vigitel_2009_preliminar_web.pdf
3. Wagner DC, Short JL. Longitudinal predictors of self-rated health and mortality in older adults. Prev Chronic Dis. 2014; 5;11:E93.

4. Lima-Costa MF, Cesar CC, Chor D et al. Self-rated health compared with objectively measured health status as a tool for mortality risk screening in older adults: 10-year followup of the Bambuí Cohort Study of Aging. Am J Epidemiol. 2012;1;175(3):228-35.

5. Tinetti ME, McAvay G, Chang SS et al. Effect of chronic disease-related symptoms and impairments on universal health outcomes in older adults. J Am Geriatr Soc. 2011; 59(9):1618-27.

6. Borim FSA, Barros MBA, Neri AL. Autoavaliação da saúde em idosos: pesquisa debase populacional no Município de Campinas, São Paulo, Brasil. Cad. Saúde Pública. 2012; 28(4):769-80.

7. Feng X, Astell-Burt T. Neighborhood socioeconomic circumstances and the co-occurrence of unhealthy lifestyles: evidence from 206,457 Australians in the 45 and up study. PLoS One 2013;15;8(8):e72643.

8. Luz TCB, César CC, Lima-Costa MF et al. Satisfaction with the neighborhood environment and health in older elderly: cross-sectional evidence from the Bambuí Cohort Study of Aging. Cad. Saúde Pública. 2011;27 Sup 3:S390-8.

9. Bowling, A. Measuring health. A review of quality of life measurement scales. Berkshire, UK: Open University Press; 2005.

10. Maia FOM, Duarte YAO, Lebrão ML et al. Fatores de risco para mortalidade em idosos. Rev. Saúde Pública. 2006; $40(6): 1049-56$

11. Hirve S. 'In general, how do you feel today?' self-rated health in the context of aging in India Global Health Action. 2014; $7: 23421$.

12. French DJ, Browning C, Kendig $\mathrm{H}$ et al. A simple measure with complex determinants: investigation of the correlates of self-rated health in older men and women from three continents. BMC Public Health. 2012;13;12:649.

13. Robert SA, Cherepanov D, Palta M et al. Socioeconomic status and age variations in health-related quality of life: Results from the National Health Measurement Study. J Gerontol B Psychol Sci Soc Sci. 2009;64B(3):378-89.

14. Crimmins EM, Kim JK, Solé-Auró A. Gender differences in health: results from SHARE, ELSA and HRS. Eur J Public Health. 2011;21(1):81-91.

15. Lima-Costa MF, de Oliveira C, Macinko J et al. Socioeconomic inequalities in health in older adults in Brazil and England. Am J Public Health. 2012;102(8): 1535-41.

16. Brenes-Camacho G. Favourable changes in economic wellbeing and self-rated health among the elderly. Soc Sci Med. 2011;72(8):1228-35.

17. Louvison MCP, Lebrão ML, Duarte YAO et al. Desigualdades no uso e acesso aos serviços de saúde entre idosos do município de São Paulo. Rev Saúde Pública. 2008; $42(4): 733-40$.

18. Hernandes ESC, Lebrão ML, Duarte YAO et al. Idosos com e sem plano de saúde e características socioepidemiológicas associadas. Rev. Saúde Pública. 2012;46(6):1030-8.

19. Lima-Costa MF, Turci MA, Macinko J. Estratégia Saúde da Família em comparação a outras fontes de atenção: indicadores de uso e qualidade dos serviços de saúde em Belo Horizonte, Minas Gerais, Brasil. Cad. Saúde Pública. 2013;29(7):1370-80. 
20. Torres JL, Dias RC, Ferreira F et al. Functional performance and social relations among the elderly in Greater Metropolitan Belo Horizonte, Minas Gerais State, Brazil: a population-based epidemiological study. Cad. Saúde Pública. 2014;30(5):1018-28.

21. Caetano SC, Silva CM, Vettore MV. Gender differences in the association of perceived social support and social network with self-rated health status among older adults: a population-based study in Brazil. BMC Geriatr. 2013; 15;13:122.

22. Szanton CL, Seplaki RJ. Thorpe Jr RJ et al. Socioeconomic status is associated with frailty: the Women's Health and Aging Studies. J Epidemiol Community Health. 2010; 64(1): 63-7.

23. Boehme S, Geiser C, Renneberg B. Functional and self-rated health mediate the association between physical indicators of diabetes and depressive symptoms. BMC Fam Pract. 2014; 20:15:157.

24. Kuh D, Ben-ShlomoY, Lynch J et al. Life course epidemiology. J Epidemiol Community Health. 2003;57(10):778.

25. Andrew AK, Keefe MK. Social vulnerability from a social ecology perspective: a cohort study of older adults from the National Population Health Survey of Canada. BMC Geriatrics. 2014;14:90.

26. Rodrigues NO, Neri AL.Vulnerabilidade social, individual e programática em idosos da comunidade: dados do estudo FIBRA, Campinas, SP, Brasil. Cien Saude Colet. 2012;17(8):2129-39.

27 Ayres J, Calazans GJ, Saletti Filho HC et al. Risco, vulnerabilidade e práticas de prevenção epromoção da saúde. In: Campos G, Minayo MCS,Akerman M, Drumond Jr M, Carvalho YM, orga-nizadores. Tratado de Saúde Coletiva. São Paulo: Editora Fiocruz; 2006. p. 375-41.

28. Maia FOM, Duarte YAO, Secoli SR et al. Adaptação transcultural do Vulnerable Elders Survey-13 (VES-13): contribuindo para a identificação de idosos vulneráveis. Rev Esc Enferm USP. 2012;46(Esp):116-2.

29. Neri AL, Yassuda MS, Araújo LFA et al. Metodologia e perfil sociodemográfico, cognitivo e de fragilidade de idosos comunitários de sete cidades brasileiras: Estudo FIBRA. Cad. Saúde Pública. 2013;29(4):778-92.
30. Fried LP, Tangen CM, Walston J et al. Frailty in older adults:evidence for a phenotype. J Gerontol A BiolSciMedSci. 2001;56:M146-56.

31. Brucki SMD, Nitrini R, Caramelli $\mathrm{P}$ et al. Sugestões para o uso do Mini-Exame do Estado Mental no Brasil. ArqNeuropsiquiatr. 2003;61:777-81.

32. Reuben DB, Laliberte L, Hiris J et al. A hierarchical exercise scale to measure function at the Advanced Activities of Daily Living (AADL) level. J Am Geriatr Soc. 1990;38: 855-61.

33. LohmanTG, Roche AF, Martorell R. Anthropometric standardization reference manual. Illinois: Human Kinetics Books 1988.

34. Almeida OP, Almeida SA. Short versions of the Geriatric Depression Scale: A study of their validity for the diagnosis of major depressive episode according to ICD-10 and DSM-IV. Int J Geriatr Psychiatry. 1999;14(10):858-65.

35. Andrade FB, Lebrão ML, Santos JLF et al. Factors related to poor self-perceived oral health among community-dwelling elderly individuals in São Paull, Brazil 2012. Cad Saúde Pública. 2012;28(10):1965-75.

36. Luz TCB, LoyolaFilho AI, Lima-Costa MF. Social capital and under-utilization of medication for financial reasons among elderly women: evidence from two Brazilian health surveys. Ciênc. Saúde Coletiva. 2013;18(12):3721-30.

37. Steptoe A, Feldman PJ, Kunz S et al. Stress responsivity and socioeconomic status: A mechanism for increased cardiovascular disease risk? Eur Heart J. 2002;23: 1757-63.

38. Carvalho MFC, Romano-Lieber NS, Bergsten-Mendes G et al. Polifarmácia entre idosos do Município de São Paulo - Estudo SABE. Rev bras epidemiol. 2012;15(4): 817-27.

39. Cassoni TCJ, Corona LP, Romano-Lieber NS et al. Uso de medicamentos potencialmente inapropriados por idosos do Município de São Paulo, Brasil: Estudo SABE. Cad. Saúde Pública. 2014;30(8):1708-20.

40. Santos TRA, Lima DM, Nakatani AYK et al. Consumo de medicamentos por idosos, Goiânia, Brasil. Rev. Saúde Pública. 2013;47(1):94-103. 